\title{
GOBERNANZA Y GESTIÓN DEL TURISMO MÉDICO EN LA REGIÓN fronteriza de Ciudad Juárez-El Paso
}

\author{
María Teresa Martínez-Almanza \\ tmartine@uacj.mx \\ Santos Alonso Morales-Muñoz \\ samorale@uacj.mx \\ Carlos Jesús González-Macías \\ cgonzalez@uacj.mx \\ Universidad Autónoma de Ciudad Juárez
}

\begin{abstract}
El objetivo de este trabajo fue profundizar en el conocimiento de la gestión del turismo médico y su relación con elementos de buena gobernanza en la región fronteriza Ciudad Juárez-El Paso, destino de gran potencial en este segmento del turismo. Mediante 30 entrevistas en profundidad a informantes clave, analizadas temáticamente, los hallazgos enfatizaron la falta de planeación, organización e integración de stakeholders para el turismo médico, y de coordinación entre los tres niveles de gobierno. Las conclusiones destacan la participación de stakeholders clave y la identificación de vacíos de poder, estancamiento del sector turístico e incipientes componentes de gobernanza.
\end{abstract}

Palabras clave: Gobernanza, turismo médico, gestión, frontera, Ciudad Juárez-El Paso.

\section{Medical Tourism Governance and Management in the Border Region of Ciudad Juarez-El Paso}

The objetive of this paper is to approach the existing relationship between medical tourism management and elements of good governance in the border region of Ciudad Juárez-El Paso, a destination of great potential in this segment of tourism. The research is based on the thematic analysis of 30 in-depth interviews with key informants. The results emphasize the lack of planning, organization and integration of medical tourism stakeholders, as well as the lack of coordination between the three levels of government. The conclusions highlight the participation of key stakeholders and the identification of power gaps, tourism sector stagnation and emerging governance components.

Key words: Governance, medical tourism, management, border, Juarez-El Paso. 
I presente trabajo tiene como propósito profundizar en el conocimiento de la gestión del turismo médico y su relación con elementos de buena gobernanza, tomando como base para el análisis los criterios propuestos por el Programa de las Naciones Unidas para el Desarrollo (PNUD) publicados en 2009 en torno a este tema y el turismo. Estos principios están asociados con la sostenibilidad de un destino turístico y son: participación, imperio de la ley, transparencia, capacidad de respuesta, orientación al consenso, equidad, eficacia y eficiencia, rendición de cuentas y visión estratégica.

La gestión de destinos se centra en administrar y apoyar la integración de diferentes recursos, actividades y agentes involucrados a través de políticas y medidas apropiadas. Conlleva, por tanto, competencias tanto gubernamentales en materia de toma de decisiones como funcionales (planificación, organización y control de actividades empresariales), que normalmente deberían incumbir al sector público (Manente, 2008).

En lo relativo al concepto de gobernanza, no obstante que ha sido empleado de diversas maneras y adopte distintos significados, existe un acuerdo general de que se trata de una nueva forma de gobernar, más cooperativa, en la cual parece que se pierden los límites entre los sectores gubernamental, privado y social. Es esa nueva gobernanza, con mayor interacción, cooperación y coordinación entre gobierno, sociedad civil y sector privado, que además supone un cambio de rumbo, debido a que el Estado ha perdido el monopolio de los recursos económicos e institucionales (Natera, 2005).

Interesa conocer el concepto de turismo médico, para el cual aún no existe consenso de los estudiosos del tema, pero para este trabajo será el siguiente: viajes médicos, turismo de salud o asistencia sanitaria global. Términos inicialmente utilizados por agencias de viaje y los medios de comunicación para describir la creciente práctica de viajar a través de las fronteras internacionales con el propósito de obtener atención médica. En términos sencillos se refiere a las personas que van a diferentes países para conseguir atención de salud (Manhas y Ramjit, 2015).

Para la realización de este trabajo, se recurrió a la consulta de una amplia fuente de recursos bibliográficos; también se contó con el apoyo de 30 stakeholders del turismo médico que incluyen autoridades de gobierno federal, estatal y municipal, directivos de hospitales, médicos cirujanos, asociaciones profesionales, sector turismo y educación, de quienes se recogieron importantes contribuciones mediante entrevistas en profundidad.

El análisis del proceso de buena gobernanza (PNUD, 2009) que se utiliza para la gestión de un destino de turismo médico en una región fronteriza significa un aporte inicial y necesario para el estudio de este tipo de fenómenos complejos y de las relaciones que de ellos se derivan. De la revisión de la literatura es posible observar que existe una incipiente producción académica sobre el tema, la cual procede principalmente de países europeos (Barbini et al., 2011). Así pues, con esta investigación se pretende establecer un punto de partida para conocer los principales lineamientos en el nivel nacional e internacional, a fin de profundizar en el conocimiento de la gobernanza en el campo turístico médico.

El trabajo se divide en seis apartados. El primero resalta la importancia de los principios de una buena gobernanza para avanzar hacia un destino turístico médico sostenible; 
el segundo se refiere a la gestión del turismo médico y la participación de los stakeholders seleccionados; el tercero presenta los métodos utilizados en la investigación; el cuarto expone los resultados; el quinto aborda la discusión; y el último ofrece las conclusiones y recomendaciones.

\section{Principios de una buena gobernanza}

La palabra gobernanza "en su uso actual no es sinónimo de gobierno; contrariamente, implica un cambio en su significado al estar referido al nuevo proceso de gobierno $o$ al nuevo método con el que se gobierna a la sociedad" (Rhodes, 2005, p. 100). Por otra parte, Peters (2003) define a la gobernanza como la capacidad presente en el gobierno para conducir la economía y la sociedad y puede señalarse como el proceso de proporcionar dirección a la sociedad.

La gobernanza es un término más extenso y vasto que el de un mero gobierno, ya que incluye y reconoce una participación activa de stakeholders privados, existiendo y permitiendo una interdependencia entre organizaciones, así como interacciones continuas entre los miembros de redes autoorganizadas en aras de intercambiar recursos y negociar objetivos compartidos y en las que si bien el Estado ya no ocupa una posición privilegiada y soberana sí puede dirigir las redes indirecta y limitadamente (Rhodes, 2005).

Para el desarrollo de una propuesta preliminar de base para su conceptualización, la Organización Mundial del Turismo (2010) establece lo siguiente:

Entendemos por gobernanza turística el proceso de conducción de los destinos turísticos a través de los esfuerzos sinérgicos y coordinados de los gobiernos en sus diferentes niveles y atribuciones, de la sociedad civil que habita en las comunidades receptoras y del tejido empresarial relacionado con la operación del sistema turístico [pp. 31-32].

Un caso de éxito digno de ser mencionado es la experiencia de Tijuana, Baja California. Las principales acciones en el periodo inicial de la transición hacia la gobernanza en Tijuana se resumen en la promoción para atraer inversiones; mejorar la coordinación público-privada; el apoyo a las micro, pequeñas y medianas empresas (mipymes), y el desarrollo urbano (infraestructura). Como resultado de un adecuado ejercicio tanto de coordinación intergubernamental como público-privada, se logró el fortalecimiento de clústeres industriales, con la finalidad de incrementar la competitividad y la economía local (Rodríguez, 2016).

Por su parte, Flores y Gómez-Sánchez (2010), en relación con la importancia que debe atribuírsele a la gobernanza en materia de salud, enuncian lo siguiente:

el análisis de la gobernanza del sistema de salud, además de tomar en cuenta el rol de los actores tradicionales en la prestación de servicios médicos y en la conducción de las políticas de salud; se debe estudiar la influencia de los gobiernos locales, los grupos organizados de la sociedad civil -de base comunitaria, sindicatos, etc.-, las instancias académicas y los agentes económicos -la industria farmacéutica, de tecnología médica, etc. [p. 145]. 
En particular algunos países en desarrollo, entre los que se encuentra México, han identificado en el turismo médico un nicho de oportunidad para la generación de beneficios económicos, donde conllevará una gran importancia en la gobernanza como proceso de toma de decisiones que afectará de forma directa e indirecta a su sistema de salud en aras de proveer la mejor atención posible, mediante acciones regidas por los principios de equidad, igualdad, solidaridad y participación (Flores y Gómez-Sánchez, 2010).

Se considera importante indagar sobre las formas de interacción de los stakeholders turísticos participantes en relación con el desarrollo del turismo médico en una ciudad fronteriza, ya que "ningún stakeholder tiene un potencial de acción suficiente para dominar de forma unilateral” (Kooiman, 2005, p. 61). El término stakeholder incluye a todas aquellas personas o grupos de personas que tienen, o reclaman, propiedad, derechos o intereses en una organización y sus actividades, pasadas, presentes o futuras, quienes desarrollan similares intereses, reclamos o derechos (Clarkson, 1995). Kooiman (2005), al igual que

Cuadro 1. Principios de buena gobernanza según el PNUD (2009)

\begin{tabular}{|c|c|}
\hline $\begin{array}{l}\text { Principios de buena } \\
\text { gobernanza }\end{array}$ & Significado \\
\hline Participación & $\begin{array}{l}\text { Todos los hombres y las mujeres deben intervenir en la toma de decisiones, ya sea } \\
\text { directamente o por medio de legítimas instituciones intermedias que representan } \\
\text { sus intereses. Esta amplia participación se basa en la libertad de asociación y ex- } \\
\text { presión, así como las capacidades para participar de manera constructiva. }\end{array}$ \\
\hline Imperio de la ley & $\begin{array}{l}\text { Los marcos legales deben ser justos y aplicados de manera imparcial, en particular } \\
\text { las leyes sobre los derechos humanos. }\end{array}$ \\
\hline Transparencia & $\begin{array}{l}\text { La transparencia se basa en el libre flujo de información. Los procesos de las ins- } \\
\text { tituciones y la información son directamente accesibles a los interesados y se les } \\
\text { proporciona lo suficiente para comprender y vigilar. }\end{array}$ \\
\hline Capacidad de respuesta & Instituciones y procesos que tratan de servir a todos los interesados. \\
\hline Orientación al consenso & $\begin{array}{l}\text { El buen gobierno interviene para lograr llegar a un consenso amplio sobre lo que es } \\
\text { el mejor interés para el grupo y, cuando sea posible, sobre las políticas y procedi- } \\
\text { mientos. }\end{array}$ \\
\hline Equidad & $\begin{array}{l}\text { Todos los hombres y las mujeres tienen oportunidades de mejorar o mantener su } \\
\text { bienestar. }\end{array}$ \\
\hline Eficacia y eficiencia & $\begin{array}{l}\text { Los procesos y las instituciones han de producir resultados que satisfagan las ne- } \\
\text { cesidades, y al mismo tiempo, hagan el mejor uso de los recursos. }\end{array}$ \\
\hline Rendición de cuentas & $\begin{array}{l}\text { Quienes toman las decisiones en el gobierno, el sector privado y las } \\
\text { organizaciones de la sociedad civil, son responsables ante el público, así como } \\
\text { ante las instituciones interesadas. Esta responsabilidad varía en función de la or- } \\
\text { ganización y si la decisión es interna o externa a la organización. }\end{array}$ \\
\hline Visión estratégica & $\begin{array}{l}\text { Los líderes y el público tienen una perspectiva amplia y a largo plazo en } \\
\text { la buena gobernanza y en el desarrollo humano, junto con un sentido de lo que se } \\
\text { necesita para dicho desarrollo. Hay también una comprensión de las complejidades } \\
\text { históricas, culturales y sociales en que se basa esa perspectiva. }\end{array}$ \\
\hline
\end{tabular}


Mayntz (2001), otorga relevancia a las relaciones entre los diferentes tipos de stakeholders como portadores de propuestas y poder para generar cambios.

La gobernanza es entendida como una forma innovadora de gobierno, distinta al antiguo modelo jerárquico, cuya estructura está compuesta por diversos stakeholders y organizaciones involucrados y las interacciones que entre ellos se generan. Además, el concepto de gobernanza permite reflexionar sobre la participación local en las políticas públicas que conciernen al turismo, tornándose necesaria su incorporación a los estudios turísticos. Para este trabajo se utilizaron los principios de buena gobernanza propuestos por el PNUD en 2009, cuyo significado se presenta en el cuadro 1.

\section{Gestión del turismo médico y participación de los stakeholders}

Una buena gestión del destino de turismo médico es concebida como un conjunto de acciones que, administrando recursos de diversa naturaleza, persigue el desarrollo turístico de un espacio o lugar de consumo determinado. En los últimos años además se considera necesario que el proceso incorpore dos principios: la implicación de todos los stakeholders interesados y la sostenibilidad (Asociación Española de Expertos Científicos en Turismo, 2001). Cualquiera que sea la propuesta conceptual sobre la gestión de destinos resulta indiscutible que los actores públicos deberían estar jugando un papel importante en ella, aunque sobre esta cuestión la investigación es aún escasa (Velasco, 2007). Los organismos de planificación y gestión turísticas se han convertido en los responsables del liderazgo, la coordinación, la promoción, la creación de productos, el marketing y el desarrollo de tales destinos, así como de la información turística que se proporciona en ellos (Ejarque y Bernet, 2005).

El problema de carecer de una buena gestión del turismo médico en la región fronteriza de Ciudad Juárez-El Paso merece ser estudiado a profundidad debido al actual desconocimiento del verdadero impacto que la ausencia de los principios de buena gobernanza puede significar, ya que esto podría obstaculizar el desarrollo de un destino de turismo médico sostenible, el cual procura dirigirse a las necesidades conjuntas de todos los actores del destino turístico (la comunidad local, el entorno físico y cultural, los negocios turísticos y los propios turistas) (Rodríguez y Fraiz, 2010) en una aproximación en la que su objetivo es el disminuir todos aquellos posibles conflictos y fricciones que se pudiesen presentar debido a la continua, pero sobre todo compleja, interacción entre los actores involucrados (Bramwell y Lane, 1993).

Cabrero (2002) destaca cuatro tipos de estrategias innovadoras para la gestación de nuevos esquemas de gobernabilidad. Se trata de una dinámica que rompe claramente con las tradiciones políticas del país, debilitando los cacicazgos locales, fragmentando los núcleos de concentración del poder y abriendo nuevos espacios para la hechura de programas y políticas en conjunto con la ciudadanía, los cuales son: un nuevo tipo de liderazgo, más abierto y horizontal; implantar nuevos mecanismos de interacción permanente entre gobierno y ciudadanos; la aparición de nuevos esquemas de relaciones intergubernamentales y 
nuevos sistemas de gestión administrativa, con experiencias de modernización altamente exitosas, como el caso de Tijuana, Baja California.

El estudio de la gestión del turismo médico a partir de los principios de buena gobernanza: participación, imperio de la ley, transparencia, capacidad de respuesta, orientación al consenso, equidad, eficacia y eficiencia, rendición de cuentas y visión estratégica, toma relevancia debido a que los habitantes de la ciudad han experimentado una etapa de crisis económica, de inseguridad, violencia e insuficiencia de servicios desde 2007, de la cual aún no ha sido posible recuperarse. Ante la creciente demanda de atención en materia de salud por parte de algunos pacientes del vecino país, el turismo médico podría convertirse en una opción de desarrollo socioeconómico. No obstante, es necesario planificar el desarrollo proveniente de la prestación de servicios de salud para que realmente se convierta en una oportunidad de generar inversión, empleos y comercio, sin perjudicar la atención médica que se oferta a la población local (Martínez-Almanza, Guía y Serra, 2017).

Ciudad Juárez aparece en la lista de destinos de turismo médico en México que promueve el gobierno federal, amén de ser conocido como un centro que oferta servicios médicos de calidad a precios razonables (ProMéxico, 2013). A pesar de las limitadas acciones realizadas para fomentar el turismo médico y de la mala reputación de la ciudad debido a la etapa de inseguridad y violencia vivida en la última década, existe una creciente demanda de servicios de salud para procedimientos específicos, que van desde la cirugía bariátrica hasta los servicios dentales especializados, además de la consulta médica general y especializada en varios hospitales y clínicas de la ciudad (Martínez, Morales y Zizaldra, 2016). Desafortunadamente, Ciudad Juárez carece de una estructura o una planificación para el desarrollo del segmento del turismo médico, dejando de explotar al máximo una de sus principales ventajas: su ubicación geográfica, que implica costos más bajos de los tratamientos, servicios de atención sanitaria más expedita, sin listas de espera para los pacientes internacionales, generación de empleos y un mayor desarrollo socioeconómico para la ciudad fronteriza (Martínez-Almaza, Guía y Serra, 2014).

Ejarque (2005) afirma que en un sistema de destino difuso los productos y los atractivos turísticos no están integrados entre sí porque no hay coordinación ni colaboración entre los productores de los servicios. La consecuencia de ello es que el destino no puede tener una personalidad clara en el mercado. Existen muchos destinos de este tipo, que desarrollan una oferta que no está organizada de ningún modo en el nivel territorial.

Debido a que el fenómeno del turismo médico, en cuanto tal, ha sido estudiado mayormente en los países y destinos de salud más exitosos, como Tailandia y Malasia, a partir de la década de los setenta, en este trabajo se ejemplifica con los países que cuentan con mayor información sobre el tema de gestión y políticas públicas para el desarrollo del turismo médico. Por ejemplo, la participación integral del Estado en la industria del turismo médico en Singapur y Malasia debe entenderse en relación con sus estrategias de desarrollo económico y el carácter del Estado. Ambas naciones heredan las instituciones estatales burocráticas bien desarrolladas al pasar a ser políticamente independientes de Gran Bretaña. Los estados poscoloniales, para su desarrollo, adoptan modelos capitalistas 
Cuadro 2. Comparación de sistemas de salud en países seleccionados

\begin{tabular}{|c|c|c|c|c|}
\hline $\begin{array}{l}\text { Elemento para el } \\
\text { análisis/ países }\end{array}$ & Tailandia & Malasia & Singapur & México \\
\hline $\begin{array}{l}\text { Estrategia } \\
\text { nacional } \\
\text { (Planeación) }\end{array}$ & $\begin{array}{l}\text { Centro Regional de } \\
\text { Salud }\end{array}$ & $\begin{array}{l}\text { Estrategia industrial } \\
\text { para desarrollar el } \\
\text { turismo }\end{array}$ & $\begin{array}{l}\text { Estrategia de creci- } \\
\text { miento económico } \\
\text { para desarrollar } \\
\text { industrias biomédi- } \\
\text { cas. Apoyo a R\&D } \\
\text { biomédico }\end{array}$ & $\begin{array}{l}\text { Promoción inter- } \\
\text { nacional a través } \\
\text { de ProMéxico y } \\
\text { certificación de } \\
\text { hospitales }\end{array}$ \\
\hline $\begin{array}{l}\text { Estructura } \\
\text { organizacional } \\
\text { (Organización) }\end{array}$ & $\begin{array}{l}\text { Centros de exce- } \\
\text { lencia en algunos } \\
\text { hospitales privados } \\
\text { Bangkok }\end{array}$ & $\begin{array}{l}\text { Creciente sector } \\
\text { privado de salud } \\
\text { por el movimiento } \\
\text { de mano de obra } \\
\text { calificada nacional }\end{array}$ & $\begin{array}{l}\text { Equilibrada mezcla } \\
\text { público-privado, el } \\
\text { sector público cor- } \\
\text { porizado }\end{array}$ & $\begin{array}{l}\text { Sistema de salud } \\
\text { mixto }\end{array}$ \\
\hline $\begin{array}{l}\text { Interacción de } \\
\text { Stakeholders } \\
\text { (Dirección) }\end{array}$ & $\begin{array}{l}\text { Agencia de la Junta } \\
\text { de Inversiones. } \\
\text { Ministerios de } \\
\text { Comercio } \\
\text { Departamento de } \\
\text { Promoción de } \\
\text { Exportaciones }\end{array}$ & $\begin{array}{l}\text { Sociedad de Ca- } \\
\text { lidad de la Salud } \\
\text { de Malasia (msoH) } \\
\text { Asociación de Hos- } \\
\text { pitales Privados de } \\
\text { Malasia } \\
\text { Asociación Médica } \\
\text { de Malasia }\end{array}$ & $\begin{array}{l}\text { Junta de Turismo } \\
\text { Ministro de Comer- } \\
\text { cio e Industria }\end{array}$ & $\begin{array}{l}\text { Impulso a la crea- } \\
\text { ción de clústeres } \\
\text { en los destinos } \\
\text { turísticos que cuen- } \\
\text { tan con recursos e } \\
\text { infraestructura para } \\
\text { el turismo médico }\end{array}$ \\
\hline $\begin{array}{l}\text { Impacto de las } \\
\text { Políticas } \\
\text { (Control) }\end{array}$ & $\begin{array}{l}\text { Problemas de cre- } \\
\text { ciente desigualdad } \\
\text { y división urba- } \\
\text { no-rural }\end{array}$ & $\begin{array}{l}\text { División público-pri- } \\
\text { vada; desigualdad } \\
\text { racial entre secto- } \\
\text { res público-privado }\end{array}$ & $\begin{array}{l}\text { Reducidos espacios } \\
\text { de diferencia en } \\
\text { ingresos entre los } \\
\text { sectores público y } \\
\text { privado }\end{array}$ & $\begin{array}{l}\text { Considerable au- } \\
\text { mento de la deman- } \\
\text { da de servicios de } \\
\text { salud privados }\end{array}$ \\
\hline
\end{tabular}

Fuente: Pocock y Phua (2011) adaptado para incluir México y elementos de gestión.

de libre mercado y priorizan el desarrollo económico en sus agendas. El cuadro 2 permite comparar los sistemas de salud, a través de las cuatro funciones de la gestión, en países seleccionados.

De acuerdo con Soto, Lutzow y González (2010), México cuenta con un sistema de salud mixto, ya que combina, en distinta proporción, cada una de las características de estos modelos. La descentralización ha sido una política clave para conciliar las exigencias de la liberalización de mercados con aquellas de la modernización del Estado en regímenes híbridos. Sin embargo, aún es notable la falta de coordinación de políticas entre los distintos niveles de gobierno ante un turismo de salud o un turismo médico que adquieren cada día una mayor relevancia (Haldenwang, 2005).

Los elementos de gestión en los tres países seleccionados en el cuadro 2 difieren en sus propósitos, prioridades y características distintivas de la política de cada uno. Pocock y Phua (2011) señalan que en Malasia, Singapur y Tailandia existe un conflicto entre los tres niveles que se basa en la definición de lo que es más importante desarrollar: la salud o la riqueza. Ya que en algunos países no se han desarrollado por completo los servicios públicos de 
salud, mientras que los privados sí lo han hecho. "Si bien el plan nacional de salud de Malasia no menciona el turismo médico como un objetivo estratégico, el Ministerio de Salud formó un Comité interministerial para la promoción del turismo médico y de salud (MNCPHT) en 2003" (Pocock y Phua, 2011, p. 5). La política del centro médico de Tailandia fue iniciada en 2003 por el gobierno y la Agencia de la Junta de Inversiones de Tailandia, en tanto que los ministerios de Comercio, el Departamento de Promoción de Exportaciones y el Ministerio de Salud, en colaboración con hospitales privados, ahora son los principales implementadores de la política (Pocock y Phua, 2011).

En cambio, en México todavía no hay una política definida con claridad para el desarrollo del turismo médico, solo acciones aisladas carentes de una planificación integral en todos los niveles. Esta situación parece existir particularmente en Ciudad Juárez, donde aún no se cubre la demanda de servicios de salud de la población local (A. Riosvelasco, comunicación personal, diciembre, 2017).

\section{Metodología}

El presente trabajo forma parte del proyecto de investigación "El turismo médico como estrategia de desarrollo para Ciudad Juárez, Chihuahua, México”, y busca obtener resultados de primera mano de los actores participantes en el desarrollo del turismo médico en esta ciudad fronteriza respecto a los elementos de gobernanza para un destino sostenible. La investigación tuvo como guía orientadora la siguiente pregunta: ¿Cómo se caracteriza la gestión del turismo médico en un destino de frontera y su relación con los principios de buena gobernanza?

El eje principal del trabajo ha sido el seguimiento de la evolución del proceso de gobernanza del destino médico fronterizo de Ciudad Juárez, Chihuahua, México; más concretamente, de las acciones realizadas por los actores gubernamentales, empresariales y los prestadores de servicios de salud, comercio y turismo que reciben visitantes de las ciudades vecinas en Estados Unidos.

El abordaje metodológico seleccionado fue exploratorio debido a que no hay muchos estudios que relacionen la gobernanza de un destino turístico médico con la frontera. Al ser un fenómeno novedoso dentro del ámbito de aplicación se requiere un marco que aporte flexibilidad para poder indagar acerca del tema. Siguiendo las ideas de Selltiz, Jahoda, Deutsch y Cook (1965) y Denzin y Lincoln (2005), este tipo de esquema proporciona tal herramienta que permite lograr un conocimiento más preciso del fenómeno a efectos de generar una base empírica.

La metodología utilizada se ubica dentro del enfoque cualitativo en tanto que busca comprender las acciones humanas y la realidad del fenómeno. El método básico elegido fue la teoría fundamentada (Charmaz, 2000; Glaser y Strauss, 1967). La selección del enfoque y de las técnicas empleadas derivó de la contextualización de la zona de estudio y de la definición de objetivos de investigación. Como podrá 
apreciarse, existen consideraciones relevantes derivadas del contexto y de las incipientes aproximaciones al estudio de la gobernanza del destino turístico fronterizo, que favorecieron la inclinación hacia un enfoque cualitativo, ya que coinciden con las ideas de Brewer y Hunter (1989), quienes mencionan la importancia que adquieren los estudios cualitativos ante la dificultad de identificar una población como objeto de estudio.

Se buscó que las personas que participaran en las entrevistas en profundidad tuvieran conocimientos y experiencia en relación con el tema de interés. Se utilizó una forma de muestreo por conveniencia, en el cual las decisiones relativas a la selección de estas personas incluyeron criterios como conocimientos especializados en el tema de investigación, la capacidad y la voluntad de los participantes que serían más propensos a aportar datos adecuados, tanto en términos de relevancia como de profundidad. Con base en el modelo de Caballero y Mugomba (2006) y Heung, Kucukusta, y Song (2010), quienes han estudiado a los stakeholders y la gestión del turismo médico, se eligieron las categorías de stakeholders mostradas en el cuadro 3.

Se ha tenido presente la aceptación de este tipo de métodos como los más convencionales y utilizados en la investigación empírica. Las entrevistas se efectuaron seleccionando a los participantes por un muestreo por conveniencia y con la técnica "bola de nieve" hasta lograr la saturación teórica, es decir, cuando los datos eran repetitivos y no había información nueva. Esto ocurrió en la entrevista número 30. Estas tuvieron una duración

Cuadro 3. Actores principales por grupo de categorías de Stakeholders

\begin{tabular}{|c|c|}
\hline Categorías de Stakeholders & Entrevistas realizadas \\
\hline Autoridades de gobierno federal & 2 \\
\hline Autoridades de gobierno estatal & 3 \\
\hline Autoridades de gobierno municipal & 5 \\
\hline Directivos de hospitales & 6 \\
\hline Médicos cirujanos especialistas & 2 \\
\hline Asociaciones profesionales & 4 \\
\hline Sector turismo & 6 \\
\hline Sector educación & 2 \\
\hline Total de entrevistas realizadas & 30 \\
\hline
\end{tabular}

Fuente: Construcción propia con base en trabajo empírico.

aproximada de 90 minutos y fueron procesadas con el software N-VIVO como apoyo para su codificación, creación de nodos y categorías de análisis para la construcción de teoría sustentando pasos y operaciones en el nivel del texto. Además, en el nivel conceptual, al definir relaciones entre códigos, categorías superiores, subcategorías y redes de categorías. 
Se tomó como base un estudio cualitativo de Casey, Crooks, Snyder y Turner (2013) sobre turismo médico. Se eligió la secuencia de pasos a seguir para el desarrollo del análisis temático de esta investigación. Esto debido a que se trata de un estudio sobre el turismo médico que también usa este tipo de análisis para la construcción de categorías; además, porque los autores mencionados han realizado valiosas aportaciones al estudio del turismo médico, utilizando el método cualitativo.

La secuencia de pasos para la realización de este trabajo fue la siguiente: todas las entrevistas fueron grabadas y transcritas, y se llevó a cabo su revisión en profundidad y notas; la identificación de unidades de análisis y extracción de significados; codificación; puesta en común con el grupo de investigadores participantes; identificación de tendencias y patrones relevantes; y, por último, la comparación con los conocimientos existentes en la literatura.

En el cuadro 4 se observan algunos de los tópicos cubiertos en las entrevistas mencionadas. Las preguntas fueron de elaboración propia con base en los principios de buena gobernanza del PNUD (2009).

Cuadro 4. Preguntas de entrevista seleccionadas

$$
\text { Pregunta Tema }
$$

1. ¿Cree usted que todos los participantes intervienen en la toma de decisiones, Participación ya sea directamente o por medio de legítimas instituciones intermedias que representan sus intereses?

2. ¿Considera usted que existen suficientes Instituciones, organismos y procesos Capacidad de respuesta que tratan de servir a todos los interesados en el desarrollo del Turismo Médico en la localidad? ¿Las que existen, tratan de servir a todos los interesados?

3. ¿Cuál es su opinión sobre la intervención del gobierno para lograr llegar a un consenso amplio sobre lo que es el mejor interés para el grupo y, cuando sea posible, sobre las políticas y procedimientos? ¿Qué ha hecho falta a este respecto?
4. ¿Considera usted que existen oportunidades de mejorar o mantener su bienestar, de manera equitativa para todas las personas que desean participar en el desarrollo del turismo médico en Ciudad Juárez?

5. ¿Cuál es su opinión sobre la obtención de resultados de los procesos y las instituciones que coordinan el turismo médico, en cuanto a la satisfacción de las necesidades de los participantes en el desarrollo del turismo médico? ¿Y en cuanto al uso de los recursos?

6. ildentifica usted la existencia de liderazgos y de actores clave que tienen un Visión estratégica sentido claro de lo que se necesita hacer para el desarrollo del turismo médico, en un marco del desarrollo humano?

7. ¿Cuál es su opinión respecto a las barreras que impiden el desarrollo del turismo Barreras para el desarrollo médico en La frontera Juárez-El Paso?

Fuente: Construcción propia con base en principios de buena gobernanza del PNUD (2009). 


\section{Resultados}

Se identificó una falta de efectividad en la gestión del turismo médico, la cual podría atribuirse a la ausencia de planificación del desarrollo de este segmento turístico en los tres niveles de gobierno. Uno de los pilares que sustentan el desarrollo de la industria del turismo médico es la normatividad y, hasta este momento, aún sigue en proceso la aprobación de la Ley de Turismo Médico propuesta por el Partido Acción Nacional (PAN) ante el Congreso. Al preguntar sobre las barreras que los actores reconocen para el desarrollo del turismo médico, las personas participantes referían una falta de planeación, integración de actores y coordinación.

El fenómeno del turismo médico es en esencia complejo. Desde la perspectiva de la oferta, intervienen varias dependencias de gobierno que pertenecen a distintas secretarías de Estado, lo que hace complicada la colaboración y la coordinación. Mientras que, del lado de la demanda, los pacientes enfrentan una realidad en la que habrán de tomar decisiones sobre la atención de su salud ante un contexto sociocultural diferente, el cual puede significar un reto para ellos. Los entrevistados aún no perciben el apoyo del gobierno federal para el desarrollo del turismo médico. A continuación, se presentan sus opiniones sobre la integración y coordinación entre los diferentes niveles de gobierno para el desarrollo del turismo médico en la frontera Juárez-El Paso:

Existe el problema del centralismo, no hay disposición del gobierno federal de entender a la zona fronteriza, no le importa. [...] No, esto no es de ahorita, ni por la violencia, ni por la inseguridad [C. Riquelme, comunicación personal, 8 de agosto, 2017].

Otros advierten una ausencia de visión estratégica y de planeación:

Se ha priorizado a la industria maquiladora para realizar estrategias que favorezcan su desarroIlo, mientras que otras opciones que requieren mayor inversión, tiempo y recursos de los que se carece, han quedado como proyectos interesantes, pero que no pasan de allí [F. Salcido, comunicación personal, 14 de noviembre, 2017].

Lo anterior refleja una falta de dirección, de liderazgo de actores, además de apoyo y financiamiento público al desarrollo de las actividades del turismo y del turismo médico, lo cual sería indispensable para el progreso de un destino turístico. Las personas entrevistadas de las distintas dependencias de gobierno de los tres niveles, quienes tenían cargos jerárquicos de primer y segundo nivel, nunca mencionaron conocer las acciones específicas para promocionar al destino médico de Ciudad Juárez, motivo por el cual se puede afirmar que existe un desconocimiento de ello. El único ejercicio plenamente identificado y señalado por varios de los entrevistados fue la creación del sistema de calidad para la certificación de hospitales, por la Secretaría de Salud.

El gobierno federal está impulsando la creación de clústeres en los estados con las características de infraestructura y recursos para ofrecer servicios de salud a pacientes internacionales. El grupo de autoridades de gobierno estatal entrevistado manifestó que se 
han realizado acciones para sensibilizar a los directivos y administradores de los hospitales privados participantes de Ciudad Juárez, en las cuales no han sido convocadas las autoridades de gobierno municipal. Por tanto, se observó una falta de coordinación entre los tres niveles de gobierno para avanzar hacia el logro de los objetivos de la política pública de turismo médico en México:

Cuando empezamos a tratar el tema del turismo médico, se percibió que todavía no llama la atención al nivel que pudiera hacerlo y esto se explica debido a que cuando los estados no tienen un desarrollo como lo tiene Chihuahua en el tema industrial, es más fácil que encaje un nuevo modelo económico que les permite desarrollar ese tema porque requieren con inmediatez de un producto para poder atraer más inversiones [F. Salcido, comunicación personal, 14 de noviembre, 2017].

Esta opinión es relevante, ya que permite advertir que el turismo médico no representa una prioridad para el gobierno del estado ni para el municipal. Es más, en varias de las entrevistas se mencionó que el municipio de Juárez no le ha otorgado al turismo médico la importancia que requiere para su desarrollo. De esta forma se explica que los apoyos existentes son orientados al reposicionamiento de la industria maquiladora. Por su parte, el gobierno municipal argumenta la ausencia de apoyo por parte del gobierno federal a la región Juárez-El Paso como causante de la falta de unión en el nivel de municipios fronterizos para la gestión de recursos y políticas públicas para el desarrollo de la frontera en el ámbito federal. Se sabe que el gobierno federal distribuye los recursos a través de los estados y, en ocasiones, estos no priorizan las necesidades de sus municipios de una manera acorde a la realidad, sino fundamentados en intereses de carácter político:

Si analizamos por qué no ha despegado el turismo médico en Juárez, yo siento que es 80 \% por cuestiones políticas y 20 \% por razones económicas [C. Flores, comunicación personal, 15 de noviembre, 2017].

Los resultados del trabajo demuestran que los aspectos de planeación e integración son determinantes en el desarrollo del destino, a través de lo cual se puede lograr un avance significativo en la consolidación del destino médico, además de favorecer el desarrollo del capital social.

En lo relativo a la participación en el clúster de turismo médico, uno de los entrevistados comentó:

Al inicio del clúster se convocaron 250 personas, de las cuales asistían 70, y posteriormente, a las reuniones de desarrollo asistían 13 en promedio. Tal vez debido a que tenían una idea errónea de que se buscaría como propósito el beneficio individual, cuando en realidad se trata de desarrollar el sector. Se busca integrar a los participantes a un proceso de calidad que no todos cumplen; en ese sentido, quizá algunos se sintieron excluidos [F. Moreno, comunicación personal, 7 de mayo, 2018].

Asimismo, el Colegio de Enfermeras de Ciudad Juárez no ha sido convocado para participar en el clúster. Estas aportaciones demuestran que los prestadores de servicios que 
atienden al turismo médico emergente no están incluidos, y que el propósito del clúster no es servir a todos los interesados, sino únicamente al grupo de prestadores de servicios que funcionan en el modelo planificado, dejando fuera a la gran mayoría de actores que trabajan en el turismo médico emergente, que reciben al grueso de pacientes de las ciudades vecinas. No se cumple el principio de participación de todos los actores involucrados propuesto por el PNUD.

En lo relativo a la capacidad de respuesta, los resultados de esta investigación demuestran que aún no existen suficientes instituciones, organismos y procesos que traten de servir a todos los interesados en el desarrollo del turismo médico en la localidad. Principalmente se destaca la débil presencia del municipio en el sector turístico y la ausencia de una institución u organismo de promoción del turismo del municipio.

En los inicios de la industria maquiladora, Tijuana era nuestro competidor, como frontera, pero luego nos unimos Juárez y Tijuana. El problema es que nos unimos en lo social pero no en lo político. Tijuana trataba de vincularse con el Distrito Federal, que no tardó en darse cuenta de la importancia de una zona de desarrollo, o una plataforma de la frontera norte como eje de desarrollo con el TLC. Por eso Tijuana tiene mayor avance y apoyo del gobierno federal [C. Riquelme, comunicación personal, 8 de agosto, 2017].

Uno de los hallazgos relevantes se refiere a que la gestión municipal del turismo no la realiza el municipio, sino el Buró de Convenciones y Visitantes de Ciudad Juárez, dependiente de un fideicomiso estatal. Las acciones municipales orientadas al desarrollo del turismo son incipientes, ya que en 2014 se creó una Dirección de Turismo, que depende de la Dirección de Promoción Económica y que promovió la creación de un Reglamento de Turismo Municipal. Esto evidencia una gran debilidad del municipio en el área de turismo, y fortalece la idea de que, en realidad, todo lo que está sucediendo en turismo médico en la ciudad obedece principalmente a la propia iniciativa privada, al gobierno del estado, al Buró de Convenciones y Visitantes, y a la comunidad emergente (quienes funcionan en el modelo teórico de turismo médico desde un marco reactivo-emergente). Lo anterior se atribuye a que el gobierno local ha priorizado el desarrollo de la industria maquiladora, por el beneficio de generación de empleos y recursos que aporta a la ciudad. Esto se explica dado que los proyectos de nueva creación como el turismo médico requieren grandes inversiones, mientras que proyectos consolidados como la industria maquiladora precisan actualmente menos recursos que cuando arrancaron. Así, se favorece a la industria maquiladora para la asignación de recursos y desarrollo de proyectos que la fortalezcan.

El desarrollo del turismo médico en la ciudad requiere el respaldo de una institución con poder, rol que el municipio aún no ha decidido asumir. Es un problema que el municipio no reconozca el valor del turismo médico para el destino turístico. Esto significa que, aunque aparentemente se está en tránsito de un proceso de desarrollo del turismo médico de emergente hacia uno planificado, en realidad han sido los mismos hospitales, clínicas y médicos que funcionan en el marco del modelo teórico del turismo médico planificado quienes realizan las acciones de planificación. 
Es inobjetable que se necesita la colaboración y apoyo tanto del gobierno del estado como del gobierno federal, pero en la medida en que el destino de turismo médico es Ciudad Juárez, consecuentemente el ente público más interesado en su desarrollo debería ser el gobierno municipal, ya que a su vez se constituye en el beneficiario directo de este proyecto.

Sería el municipio el que debería liderar estos procesos, o apoyar lo que ya existe. Pero no puede permanecer al margen. Una de las personas entrevistadas del grupo de facilitadores mencionó:

El turismo médico genera nueve empleos por operador a diferencia de la industria maquiladora que únicamente genera 2.7 empleos por cada uno. Este dato parece que no se ha dimensionado en cuanto a las posibilidades de desarrollo que podría traer a la región [S. Máynez, comunicación personal, 5 de noviembre, 2017].

En una ciudad que tradicionalmente ha fundamentado gran parte de su desarrollo económico en la industria maquiladora, se hace más difícil que se visualice un proyecto por el turismo. Es una competencia entre sectores, y la atención que estos reciben de los tres niveles de gobierno. Por tanto, falta visión estratégica, planeación y coordinación en el nivel de sectores y áreas económicas.

CuAdro 5. Elementos de gobernanza del PNUD identificados en el destino médico fronterizo de Ciudad Juárez - El Paso

\begin{tabular}{|c|c|c|c|}
\hline \multicolumn{4}{|c|}{$\begin{array}{l}\text { Elementos de un buena gobernanza en Ciudad Juárez } \\
\text { para el Turismo Médico }\end{array}$} \\
\hline Criterios del PNUD (2009) & $\mathrm{Si}$ & No & Incipiente \\
\hline Participación & & & $x$ \\
\hline Imperio de la ley & $x$ & & \\
\hline Transparencia & & $X$ & \\
\hline Capacidad de respuesta & & & $x$ \\
\hline Orientación a consenso & & $x$ & \\
\hline Equidad & & $X$ & \\
\hline Eficacia y eficiencia & & & $x$ \\
\hline Rendición de cuentas & & $x$ & \\
\hline Visión estratégica & & $x$ & \\
\hline
\end{tabular}

Fuente: Construcción propia con base en trabajo empírico. 


\section{Discusión}

La discusión sobre los hallazgos de las entrevistas realizadas a los actores participantes en el turismo médico de Ciudad Juárez se basa en teorías que sugieren que un destino turístico médico que lleva a cabo una buena gestión que incluya elementos de gobernanza tiene mayor probabilidad de cumplir sus objetivos de desarrollo.

El estudio de caso de Ciudad Juárez destaca un gran interés de los participantes en el desarrollo de la industria del turismo médico, no obstante, la forma de conducir las actividades económicas y los grupos sociales difieren con los conceptos de Rhodes (2005), quien enfatiza que en la gobernanza existe una nueva forma de gobierno mucho más participativa; aspecto que todavía no se identifica en el caso de estudio. También se observó que, en sentido opuesto a la definición de Peters (2003), existe una limitada capacidad del gobierno para proporcionar dirección a la sociedad ante una industria turística médica en pleno auge, cuya demanda de servicios por parte de pacientes del vecino país crece más cada día.

La información obtenida de los stakeholders participantes, expertos en políticas de salud, administración de la atención médica, y los sectores de turismo y educación, enfatiza la falta de planeación e integración de actores para el turismo médico. Este aspecto difiere del concepto de gobernanza propuesto por la Organización Mundial del Turismo (2010), que hace referencia al proceso de conducción de los destinos turísticos a través de los esfuerzos sinérgicos y coordinados de los gobiernos en sus diferentes niveles y atribuciones, de la sociedad civil que habita en las comunidades receptoras y del tejido empresarial relacionado con la operación del sistema turístico. En el caso del Hospital de la Familia -ubicado en el centro de la ciudad, justamente al atravesar una calle del cruce internacional Santa $\mathrm{Fe}-$, los entrevistados mencionaron el interés por continuar ofreciendo servicios de salud a pacientes del país vecino, e incluso señalaron contar con un plan estratégico de largo alcance, que incluye formación de recursos humanos para el turismo médico, para lo cual se construyó una escuela de enfermería con formación que incluye dominio del idioma inglés. Se observó una clara orientación al tratamiento de pacientes extranjeros para maximizar sus ganancias, sin embargo, este hospital no participa en el clúster de turismo médico, debido a que atiende pacientes de escasos recursos de ambos lados de la frontera y no ha sido convocado a tomar parte en las iniciativas de desarrollo del turismo médico, pese a que recibe en promedio 30000 pacientes al año provenientes de las ciudades cercanas en Estados Unidos.

A Ciudad Juárez se le presenta el reto de aprender de la experiencia de éxito de otras ciudades fronterizas en lo relativo a la gobernanza, por ejemplo Tijuana, Baja California. Rodríguez (2016) afirma que esta ciudad ha tenido periodos de administración municipal con muy buenos resultados sobre una base de planeación y de la promoción para atraer inversiones; mejorar la coordinación público-privada; el apoyo a las mipymes, y el desarrollo urbano (infraestructura). Como producto de un adecuado ejercicio tanto de coordinación intergubernamental como público-privada, se logró el fortalecimiento de clústeres industriales, con la finalidad de incrementar la competitividad y la economía local. 
La mayoría de los participantes en las entrevistas habló negativamente sobre las formas de gestión del turismo médico en Ciudad Juárez: se identifica una falta de planeación, organización, dirección y control en todos los niveles de gobierno, además de una ausencia de coordinación e integración de actores; esto se considera trascendente debido a que en 2014 aún no se contaba en el municipio con un reglamento de turismo y tampoco existe en la actualidad una dependencia municipal que promueva, organice y planifique las actividades turísticas, pese a la relevancia que estas tienen. Las funciones de promoción las realiza el Buró de Convenciones y Visitantes, dependiente del gobierno del estado, lo que nos lleva a advertir que no se cumplen los roles de los actores tradicionales, ni mucho menos se tiene la apertura para dirigir y motivar a los actores hacia una participación y compromiso con el desarrollo de la industria de este segmento del turismo. La influencia del gobierno local no se percibe y faltan liderazgos políticos que impulsen la gobernanza en materia de salud mencionada por Flores y Gómez-Sánchez (2010).

De tal suerte, se puede apuntar que el ente encargado de la gestión del turismo y del turismo médico en Ciudad Juárez es el Buró de Convenciones y Visitantes, que no tiene en cuenta el principio de participación, ya que parte de la gestión del turismo médico se lleva a cabo por empresarios que invierten en recursos turísticos médicos del destino, como la construcción de los hospitales Ángeles y Star Médica, con una limitada participación de los actores locales. El control y el poder están en manos de empresas e instituciones de fuera de la zona, creando malestar social entre los stakeholders locales.

En este sentido, no se cumple con lo establecido en el proceso que recomiendan Kooiman (2005) y Mayntz (2001) al hablar de una orientación institucional técnicamente estructurada, basada en principios, normas, procedimientos y prácticas para decidir de manera colectiva sobre objetivos comunes de convivencia y cómo coordinar y cooperar para el logro de los objetivos planteados. Asimismo, se señala que la rendición de cuentas del organismo de gestión es deficitaria, por lo que esta entidad no cumple con los principios de buena gobernanza en su gestión.

Las barreras para el desarrollo del turismo médico identificadas son similares a las que indican algunos autores, por ejemplo Keyser (2002), quien planteó que el restringido acceso a los mercados financieros, la escasa confianza por parte de los inversores internacionales y nacionales, los requisitos y los procedimientos tributarios complicados, la asignación presupuestaria limitada, la falta de integración y de promoción turística son elementos que inhiben el desarrollo del turismo en una región en particular.

En el caso de Tijuana, se considera relevante el resultado de un adecuado ejercicio tanto de coordinación intergubernamental como público-privada. Los elementos de éxito en la gestión de esta ciudad fronteriza se advierten precisamente como algunas de las barreras que han impedido el desarrollo del turismo médico en Ciudad Juárez, como la falta de planeación y de promoción para atraer inversiones, de la mejora en la coordinación público-privada, de apoyo a las mipymes, y el desarrollo urbano (infraestructura).

Las limitaciones de gestión del sector identificadas en las entrevistas refieren un plan de implementación inadecuado, ya que la creación del clúster de turismo médico incluye 
solo a los grandes hospitales y médicos ubicados en infraestructura de alto standing, los stakeholders participantes en el turismo médico emergente han desplegado sus propias estrategias individuales y carecen de una planeación del desarrollo del sector. Además, se advirtió una capacidad institucional deficiente, lo cual habla de problemas de organización, falta de rendición de cuentas y limitada capacidad de respuesta (véase figura 1).

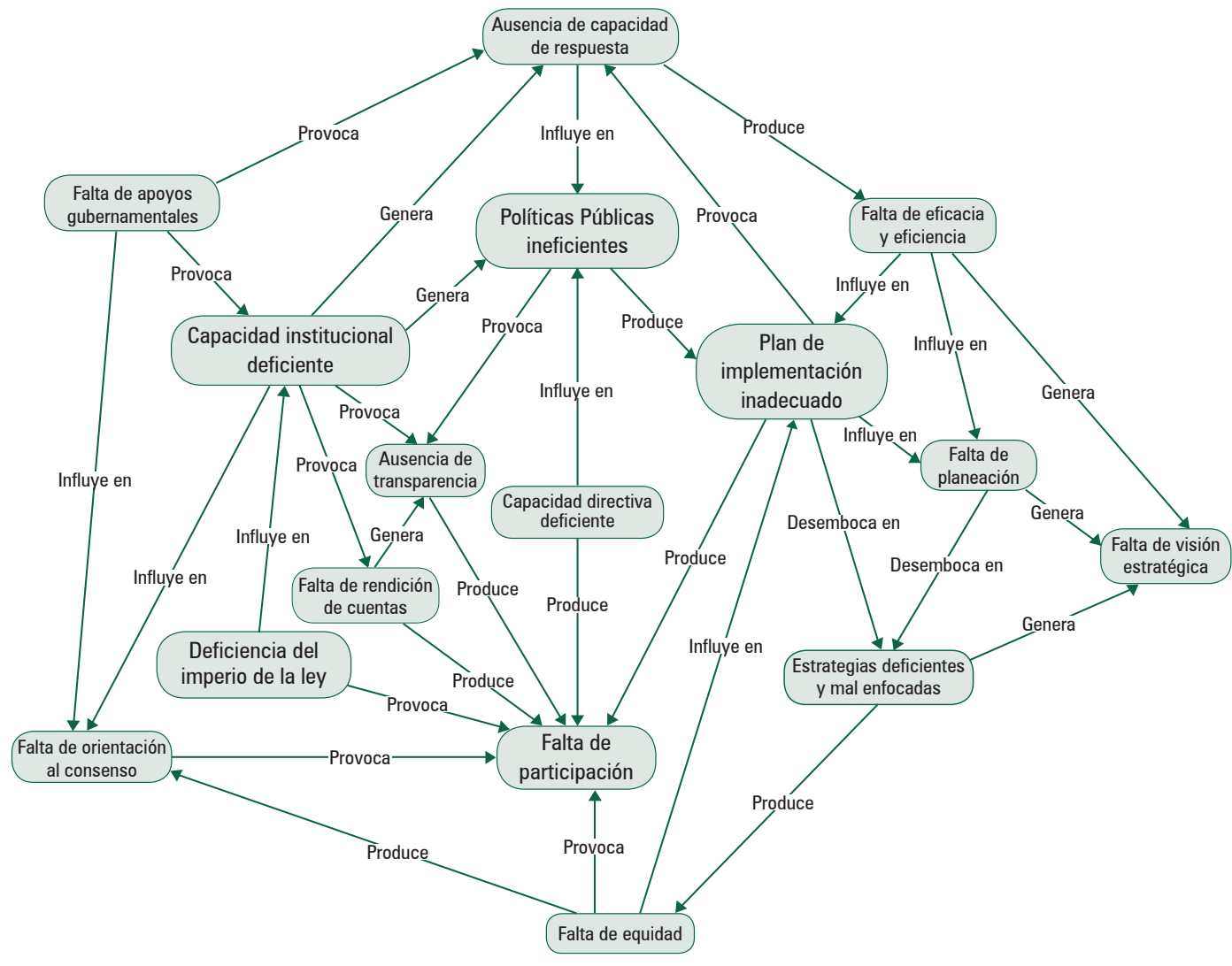

Figura 1. Red Semántica de Elementos de Gestión y Gobernanza en Ciudad Juárez

En esta investigación se ha encontrado información sobre el apoyo del gobierno de México, con acciones de promoción a través de ProMéxico y con la creación del sistema de calidad para la certificación de hospitales por medio de la Secretaría de Salud. Sin embargo, todo parece indicar que aún no ha decidido apostar por el turismo médico como estrategia de desarrollo con acciones más determinantes, a diferencia de Singapur y Tailandia, países que destacan por sus políticas y buena gestión del turismo médico.

Pocock y Phua (2011) afirman que el turismo médico ofrece una oportunidad para la coordinación de políticas entre ministerios de Salud, de Comercio y de Turismo. Se refiere a la experiencia de coordinación en Tailandia, Singapur y Malasia, en donde se está llevando a cabo una aparente convergencia en las prioridades comerciales, de turismo y del 
Ministerio de Salud, lo cual refleja la creciente aceptación de la salud como un bien privado en el ámbito mundial. En este caso de estudio, los descubrimientos indican la existencia de una escasa coordinación entre los distintos niveles de gobierno. Incluso los actores del sector privado parecían tener mayor conocimiento de las acciones realizadas por el gobierno federal que los actores del sector público.

Heung et al. (2010) destacan la importancia de la coordinación entre las instituciones médicas y los hoteles para el periodo de recuperación de los pacientes luego de una cirugía. Empero, en Ciudad Juárez aún no se observa este tipo de coordinación, quizá debido a que los pacientes que requieren permanecer algunos días después del tratamiento médico prefieren hospedarse en la vecina ciudad de El Paso, Texas, en Estados Unidos. Por otra parte, la existencia de hospitales especialmente construidos para la atención del turismo médico reduce la necesidad del servicio de hostelería, dado que la gente se hospeda en el mismo hospital, que tiene características de un hotel de lujo. Además, en la revisión de literatura no se encontraron referencias en las que se mencione un destino turístico médico que brinde un servicio integrado en una ciudad región, donde la atención sanitaria se ofrezca en un sitio y los servicios complementarios como el transporte aéreo y el hospedaje se oferten del otro lado de la frontera. Por lo anterior, se considera que la aportación del caso empírico de la región fronteriza Ciudad Juárez-El Paso a la literatura es de gran relevancia.

También Heung et al. (2010) sostienen que los productos de turismo médico combinan los componentes de la salud y el turismo. No obstante, los hallazgos del caso de estudio revelan elementos distintos en el sentido de que el producto va a la prestación de servicios de salud, donde la coordinación entre los sectores de la salud y el turismo es escasa. Probablemente debido a que son pocos los atractivos turísticos, y los que existen están poco desarrollados. Hay ausencia de un organismo potente con respaldo institucional para gestionar el turismo en la localidad.

En cuanto al liderazgo, Cabrero (2002) pone como ejemplo el caso exitoso de Tijuana, Baja California, como una de las estrategias innovadoras de gobernanza local que basa su acción en un nuevo tipo de liderazgo, más abierto y horizontal, que promueve acciones de trabajo en equipo en programas y políticas conjuntamente con la ciudadanía. En cuanto a Ciudad Juárez, existen iniciativas como el Plan Estratégico de Juárez, que tiene por objetivo la construcción de una fuerza ciudadana que participe en los asuntos públicos. Sin embargo, todavía no se aprecian resultados tangibles de sus acciones.

\section{Conclusiones y recomendaciones}

La presente investigación contribuye a la literatura del turismo médico mediante un examen crítico a las iniciativas de la política gubernamental para el desarrollo sostenible de la industria del turismo médico en México, identificando una brecha significativa en la región fronteriza de Ciudad Juárez-El Paso en materia de gestión y elaboración de políticas sobre turismo médico, concluyendo así que existe una incipiente aplicación de los criterios de 
buena gobernanza propuestos por el PNUD en la gestión del turismo médico en la frontera Juárez-El Paso.

En cuanto a la participación en la toma de decisiones, se concluye que se está aplicando este principio de manera incipiente, dado que algunas de las personas entrevistadas argumentan no haber sido convocadas aún para participar en el clúster de turismo médico.

Se cuenta con pleno conocimiento de las necesidades que presentan los pacientes del extranjero que pueden solicitar servicios de salud en México. Esto se puede afirmar con base en las acciones del gobierno federal de México para favorecer el desarrollo del turismo médico, que, hasta el momento de esta investigación, han sido meramente de promoción de los destinos y la creación del sistema de calidad para la certificación de hospitales, planteando la conjetura de que esto puede deberse a la previsión del impacto del desarrollo del turismo médico a gran escala en el sistema de salud del país, el cual enfrenta en la actualidad una realidad social compleja.

Es de esperarse que la demanda de servicios de salud por parte de pacientes del extranjero continúe al alza, ya que los gobiernos de los países desarrollados y empleadores están viendo cada vez más el turismo médico como una opción para reducir los costos derivados de atención en salud pública, no solo los pacientes con seguro médico público o privado sino también el paciente consumidor que carece de seguro y paga los servicios de su propio bolsillo (Wendt, 2012).

En cuanto a aspectos de integración de stakeholders, el papel mediador de la coordinación y colaboración establecido en la literatura, al igual que el impacto de la integración en los productos y atractivos turísticos, es muy relevante, aunque desafortunadamente aún hay un largo camino por recorrer en la región fronteriza, debido a la existencia de un sistema de destino difuso donde los productos y los atractivos turísticos no están integrados entre sí gracias a la ausencia de coordinación y colaboración entre los productores de los servicios, por lo que el destino no puede tener una personalidad clara en el mercado, ni desarrollar una oferta organizada de ningún modo en el nivel territorial (Ejarque, 2005).

Estas características se identificaron en el modelo de turismo médico emergentereactivo, donde la evolución de la oferta de servicios se relaciona con el creciente comportamiento de la demanda, pero sin coordinación ni colaboración de los stakeholders. Por tanto, se recomienda que las instituciones que cuentan con un liderazgo grupal orienten sus acciones al fortalecimiento de la colaboración entre los actores participantes.

De la misma manera, fue posible comprobar para el modelo de turismo médico planificado la importancia del papel mediador de los operadores externos al destino o facilitadores. Para este tipo de turismo médico se advirtió la existencia de un sistema de destino donde la integración, desarrollo de los productos y su propuesta de valor están en manos de operadores externos, o bien, de los turoperadores. El organismo de gestión turística no puede entonces desarrollar su papel de coordinación y promoción de la oferta debido a la falta de autonomía y autoridad sobre el producto, y, en consecuencia, no puede ofrecer un valor añadido propio. Se podría especular que, en el caso de los prestadores de servicios de turismo médico que contratan los servicios de facilitadores externos, puede llegar a generarse este tipo de dependencia. 
Por otra parte, en la forma de operación del turismo médico en Ciudad Juárez se identifica la ausencia de una organización que gestione el destino (Destination Marketing Organization, DMo) en el nivel municipal, afectando los resultados de su evolución. El turismo médico que existe en la ciudad ha sido promovido principalmente por los propios hospitales y médicos especialistas, develando una escasa coordinación tanto entre organizaciones privadas como públicas.

Se reconoció que la asociatividad existente entre los proveedores de servicios de salud con los de turismo y hostelería es esencial para brindar una experiencia satisfactoria al paciente que espera un servicio integral. En este sentido, el destino médico aún presenta retos importantes a superar, dado que no se localizó evidencia de acciones de cooperación o asociación entre organizaciones a favor del turismo médico; en cambio, emergió un aspecto positivo referente a la segmentación del mercado. Los prestadores de servicios de salud han podido desarrollar un enfoque centrado en las necesidades del paciente consumidor para la comercialización, el producto y la prestación del servicio, acorde con el perfil del paciente que reciben. Por ejemplo, el Hospital de la Familia atiende más de 30 000 pacientes transfronterizos al año, los cuales buscan un servicio de calidad estándar y cuya satisfacción se demuestra con las cifras de pacientes atendidos, que aumentan en miles cada año. El Centro Médico de Especialidades atiende pacientes en consulta de especialistas reconocidos, que, por su calidad, se han ganado su lealtad desde hace más de 60 años; además de los dos nuevos hospitales que cuentan con la infraestructura y estrategias de marketing que les permiten ofrecer servicios que en ocasiones superan las expectativas de los pacientes extranjeros que los visitan, principalmente por cirugía bariátrica y estética. No obstante, los hospitales carecen de una coordinación entre ellos. Cada uno desarrolla sus propias estrategias, lo cual habla de una falta de planeación, dirección y visión estratégica del destino.

En relación con el liderazgo se concluye que en el destino médico fronterizo hay diferentes liderazgos individuales, los cuales no trabajan de manera conjunta con un fin común. En virtud de que un elemento muy importante de la integración-coordinación es la presencia de liderazgo y de un sistema de gestión sistémico, se torna deseable fomentar la participación y generación de compromisos que ayuden a identificar y consolidar liderazgos de grupo que faciliten el avance en los procesos de desarrollo del turismo médico. Asimismo, se develó la existencia de un problema de falta de arraigo que ha impedido la presencia de liderazgos tanto singulares como sistémicos.

Por lo anterior se concluye que en Ciudad Juárez aún falta un largo camino por recorrer para acercarse a los criterios y recomendaciones para una buena gobernanza propuestos por el PNUD en lo referente a la gestión del turismo médico. 


\section{Fuentes consultadas}

Asociación Española de Expertos Científicos en Turismo. (septiembre, 2001). Nuevas tendencias de ocio y turismo: su especial problemática en destinos singulares. VI Congreso AECIT, Ceuta.

Barbini, B., Biasone, A., Cacciutto, M., Castellucci, D., Corbo, Y. y Roldán, N. (octubre, 2011). Gobernanza y turismo: Análisis del estado del arte. Simposio Internacional Gobernanza y Cambios Territoriales: Experiencias Comparadas de Migración de Amenidad en Las Américas (pp. 111-125). Pucón, Chile.

Bramwell, B. y Lane, B. (1993). Sustainable tourism: An evolving global approach. Journal of Sustainable Tourism, 1(1), 1-5.

Brewer, J. y Hunter, A. (1989). Multimethod Research: A Synthesis of Styles. Newbury Park: Sage.

Caballero-Danell, S., y Mugomba, C. (2007). Medical Tourism and its Entrepreneurial Opportunities-A conceptual framework for entry into the industry. Tesis de Maestría núm. 2006: 91. School of Business, Economics and Law. Gotermburgo, Suecia: Göteborg University.

Cabrero, E. (2002). El premio gobierno y gestión local en México : un mecanismo de institucionalización de la innovación en gobiernos locales. VII Congreso Internacional del CLAD sobre la Reforma del Estado y de la Administración Pública (pp. 1-11).

Casey, V., Crooks, V. A., Snyder, J., y Turner, L. (2013). Knowledge brokers, companions, and navigators: a qualitative examination of informal caregivers' roles in medical tourism. International Journal for Equity in Eealth, 12(1), 94.

Charmaz, K. (2000). Grounded theory: Objectivist and constructivist methods. Handbook of Qualitative Research, 2, 509-535.

Clarkson, M. (1995). A stakeholder framework for analyzing and evaluating corporate social performance. Academy of Management Review, 20, 92-117.

Denzin, N. y Lincoln, Y. (2005). Handbook of Qualitative Research. Thousand Oaks: Sage.

Ejarque, J. (2005). Destinos turísticos de éxito. Diseño, creación, gestión y marketing. Madrid, España: Pirámide.

Ejarque, J. y Bernet, J. E. (2005). Destinos turísticos de éxito. Madrid: Grupo Anaya Comercial, España.

Flores, W. y Gómez-Sánchez, I. (2010). La gobernanza en los Consejos Municipales de Desarrollo de Guatemala: Análisis de actores y relaciones de poder. Revista de Salud Pública, 12, 138-150.

Glaser, B. y Strauss, A. (1967). The discovery of grounded theory: Strategies for qualitative research. Chicago: Aldine.

Haldenwang, C. (abril, 2005). Gobernanza sistémica y desarrollo en América Latina. Revista de la CEPAL, 85, 35-52.

Heung, V., Kucukusta, D. y Song, H. (2010). A conceptual model of medical tourism: Implications for future research. Journal of Travel \& Tourism Marketing, 27(3), 236-251. Recuperado de http://doi.org/10.1080/10548401003744677

Keyser, H. (2002). Tourism Development. Cape Town: Oxford University Press. 
Kooiman, J. (2005). Gobernar en gobernanza. En A. Cerrillo i Martínez (coord.), La gobernanza hoy: 10 textos de referencia (pp. 57-82). Madrid: Instituto Nacional de Administración Pública.

Manente, M. (2008). Gestión de destinos y antecedentes económicos : definición y supervisión de destinos turísticos locales. Documento principal, Sesión 4: Gestión de destinos turísticos. Recuperado de http://cf.cdn.unwto.org/sites/all/files/pdf/ manente_sp.pdf

Manhas, P. y Ramjit., M. (2015). Marketing analysis of medical tourism in India. Enlightening Tourism, 5(1), 1-39.

Martínez, M., Morales, A. y Zizaldra, I. (diciembre, 2016). El turismo médico: tendencia de transformación para Ciudad Juárez, México. TURyDES, Turismo y Desarrollo Local, 21, 1-14.

Martínez-Almanza, M., Guía, J. y Serra, C. (noviembre de 2014). Desarrollo de un destino de turismo médico transfronterizo en Ciudad Juárez, México desde una perspectiva relacional. En Innovación y Competitividad en Áreas Turísticas: Actas del Seminario Internacional "Innovación y competitividad en Áreas Turísticas (ICAT2014)". 5, 6 y 7 de noviembre de 2014. Alicante, España: Instituto de Investigaciones Turísticas de Alicante.

Martínez-Almanza, M., Guía, J. y Serra, C. (2017). Génesis y evolución del turismo médico en Ciudad Juárez, Chihuahua, México. En Tendencias del turismo en Latinoamérica (pp. 176-196). Barranquilla, Colombia: Editorial Uniautónoma, Universidad Autónoma del Caribe,.

Mayntz, R. (2001). El Estado y la sociedad civil en la gobernanza moderna. Reforma y Democracia, 21(21), 7-22.

Natera, A. (2005). La gobernanza como modo emergente de gobierno y gestión pública. Gestión y Análisis de Políticas Públicas, (33-34).

Organización Mundial del Turismo (2010). Proyecto de gobernanza para el sector turismo. Informe Ejecutivo. Estadísticas y Cuentas Satélite de Turismo. Madrid: Autor.

Peters, B. (octubre, 2003). La capacidad para gobernar: ¿retrocediendo hacia el centro? Reforma y Democracia, 27. Recuperado de http://www.clad.org/portal/ publicaciones-del-clad/revista-clad-reforma-democracia/articulos/027-octubre-2003/0047218

PNUD. (2009). Good Governance and Sustainable Human Development. Recuperado de http://mirror.undp.org/magnet/policy/chapter1.htm

Pocock, N. y Phua, K. (2011). Medical tourism and policy implications for health systems: A conceptual framework from a comparative study of Thailand, Singapore and Malaysia. Globalization and Health, 7(12), 1-12.

ProMéxico. (2013). PROMEXICO Turismo Médico. Recuperado de http://mim.promexico. gob.mx/work/sites/mim/resources/LocalContent/374/2/130925_DS_Turismo_de_salud_ESP.pdf

Pulido-Fernández, M. D. L. C. y Pulido-Fernández, J. I. (2014). ¿Existe gobernanza en la actual gestión de los destinos turísticos? Estudio de casos. PASOS. Revista de Turismo y Patrimonio Cultural, 12(4), 685-705.

Rhodes, R. (2005). La nueva gobernanza: gobernar sin gobierno. La Gobernanza Hoy, 10, 99-122. 
Rodríguez, D. y Fraiz, J. (2010). Gestión de crisis en el turismo: La cara emergente de la sostenibilidad. Tourism \& Management Studies, 6, 49-58. Recuperado de http:// www.scielo.gpeari.mctes.pt/pdf/ec/n6/n6a06.pdf

Rodríguez, G. (2016). Gobernanza para el desarrollo local: el caso del Municipio de Tijuana 2007-2013. Tlatemoani, Revista Académica de Investigación, 21, 100-124.

Selltiz, C., Jahoda, M., Deutsch, M. y Cook, S. (1965). Métodos de investigación en las relaciones sociales. Madrid: Ediciones RIALP.

Soto, G., Lutzow, M. A. y González, R. (2010). Rasgos generales del sistema de salud en México. En La salud pública y el trabajo en comunidad (pp. 150-167). México: McGraw-Hill.

Velasco, M. (2007). Gestión de destinos: igobernabilidad del turismo o gobernanza del destino ? Ministerio de Educación y Ciencia.

Wendt, K. (2012). Medical Tourism: Trends and Opportunities (Tesis de maestría). University of Nevada, Las Vegas. 